\title{
Evento Científico sobre Meditação e Mindfulness no Brasil: Relato de Experiência
}

\author{
Carolina Baptista Menezes ${ }^{1}$ \\ Universidade Federal de Pelotas, Pelotas, RS, Brasil
}

\section{Resumo}

As práticas meditativas vêm sendo crescentemente estudadas, adaptadas e aplicadas no ocidente, especialmente no campo da ciência psicológica. Apesar da área já ter adquirido expressiva disseminação e visibilidade no que tange à sua produção científica mundial, no Brasil este ainda é um campo incipiente. O objetivo deste relato é divulgar e descrever dois eventos científicos conjugados sobre meditação e mindfulness ocorridos no ano de 2014 em São Paulo, Brasil, dos quais tive a oportunidade de participar na qualidade de mediadora, palestrante e ouvinte. Além de uma contextualização e caracterização geral dos eventos, este relato explora alguns temas apresentados, em especial aqueles com relevância para a ciência psicológica. De forma conjunta, as atividades desenvolvidas no Pré-Simpósio e Simpósio permitiram uma rica reflexão e discussão acerca de questões científicas e clínicas pertinentes e atuais, contribuindo para o crescimento, o reconhecimento e o avanço da área no Brasil. Espera-se que cada vez mais eventos sobre o tema sejam criados e realizados no território nacional, especialmente visando que o estudo sobre meditação/mindfulness seja formalizado enquanto área da ciência psicológica Brasileira.

Palavras-chave: Meditação, mindfulness, relato de experiência, ciência psicológica.

\section{Scientific Event on Meditation and Mindfulness in Brazil: An Experience Report}

\begin{abstract}
Meditation practices have been increasingly studied, adapted and applied in the west, particularly in the field of psychological science. Despite the fact that the area has already conquered expressive dissemination and visibility regarding its global scientific production, this is still an incipient field in Brazil. This report aimed to inform about and describe two interrelated scientific events about meditation and mindfulness that took place in the year of 2014 in São Paulo, Brazil, and of which I had the opportunity to take part as mediator, lecturer and participant. In addition to generally contextualizing and characterizing both events, this report explored some of the themes presented, especially those relevant to psychological science. Taken together, activities carried out in both events allowed a rich discussion and reflection concerning scientifically and clinically relevant issues, contributing to the rise, acknowledgment and advancement of the area in Brazil. It is hoped that more events about this theme are created and held in national territory, especially aiming that the study of meditation/mindfulness becomes formalized as a Brazilian psychological scientific area.
\end{abstract}

Keywords: Meditation, mindfulness, experience report, psychological science.

Endereço para correspondência: Universidade Federal de Pelotas, Faculdade de Psicologia, Av. Duque de Caxias, 250, Pelotas, RS, Brasil 96030-001. Fone: 53 3921-6992. E-mail: menezescarolina@hotmail.com Atualmente a autora é Professora do Programa de Pós-Graduação em Psicologia da Universidade Federal de Santa Catarina (UFSC). 


\section{Evento Científico sobre la Meditación y Mindfulness en Brasil: Relato de Experiência}

\section{Resumen}

Las prácticas de meditación han sido cada vez más estudiado, adaptado y aplicado en Occidente, especialmente en el campo de la ciencia psicológica. Aunque ha adquirido significativa difusión y visibilidad con respecto a su producción científica mundial, en Brasil este campo es todavía naciente. El objetivo de este informe es difundir y describir dos eventos científicos combinados acerca de la meditación y mindfulness ocurridos este año en São Paulo, Brasil, de los cuales tuve la oportunidad de participar como mediador, conferencista y oyente. Además de una general caracterización y contextualización de los eventos, este informe explora algunos de los temas presentados, especialmente los que tienen relevancia para la ciencia psicológica. En conjunto, las actividades de los eventos han permitido una rica reflexión y discusión de temas científicamente y clínicamente relevantes y actuales, contribuyendo al crecimiento, reconocimiento y promoción de este campo de estudio en Brasil. Se espera que más eventos sobre el tema sean creados y realizados en el país, especialmente con el objetivo de que el estudio de la meditación / mindfulness sea formalizado como un área de la ciencia psicológica Brasileira.

Palabras clave: Meditación, mindfulness, relato de experiência, ciencia psicológica.

\section{Meditação, Mindfulness e Psicologia}

Tem havido um crescente interesse no estudo científico da meditação, particularmente em relação ao seu potencial clínico no campo da saúde mental (Menezes \& Dell'Aglio, 2009; Wallace \& Shapiro, 2006). Neste contexto, a meditação tem sido descrita como uma forma de treinamento mental que visa desenvolver habilidades autorregulatórias, tanto ligadas aos domínios emocional e social, como cognitivo (Chiesa \& Malinowski, 2011; Lutz, Slagter, Dunne, \& Davidson, 2008). Operacionalmente, a meditação pode ser caracterizada a partir de um conjunto de cinco parâmetros: (a) deve ser uma técnica claramente definida; (b) deve-se alcançar algum relaxamento físico ao longo do processo; (c) deve-se alcançar algum relaxamento da lógica ao longo do processo; (d) deve ser um estado autoinduzido; e (e) deve haver a utilização de algum tipo de foco atencional (Cardoso, Souza, Camano, \& Leite, 2004). Quando o foco (ou âncora) da meditação é pontual e específico, tal como a repetição de um som ou palavra, descreve-se a prática como concentrativa, podendo também ser chamada de meditação da atenção focada. Já a utilização de um foco aberto e vigi- lante, tal como a observação não reflexiva e não associativa do próprio fluxo mental, caracteriza o subtipo meditativo mindfulness, o qual também pode ser designado de meditação do monitoramento aberto (Lutz et al., 2008).

Um estudo de revisão sistemática e meta-análise com amostras saudáveis (Sedlmeier et al., 2012) apontou que a prática de meditação ("concentrativa", "mindfulness", e "outros tipos" - abarcando a meditação guiada e aquelas sem uma descrição clara do método) está especialmente relacionada a ganhos psicológicos, com tamanhos de efeito moderados.

Dentre os principais resultados apontados pela revisão, destacam-se a redução de afeto negativo, de sintomas de estresse e ansiedade, a melhora de parâmetros atencionais, assim como o aumento dos níveis de bem-estar. Já com amostras clínicas, uma revisão sistemática apontou que os resultados parecem menos robustos (Goyal et al., 2014). Para estas amostras, os efeitos foram moderados principalmente para sintomas de ansiedade, de depressão e de dor, sendo que para variáveis de qualidade de vida, humor positivo, atenção e sono os efeitos foram considerados baixos.

A implicação terapêutica destes efeitos psicológicos, aliada à crescente busca por evidên- 
cias empíricas, tem conferido à meditação uma posição de destaque, tornando-a protagonista de tratamentos complementares em contextos de saúde mental (Chiesa \& Serretti, 2011) e saúde física (Grossman, Niemannb, Schmidtc, \& Walach, 2004). Embora extensamente aplicada no formato grupal por meio de programas, cabe destacar que a prática também tem sido progressivamente incorporada ao repertório de técnicas das psicoterapias individuais (Davis \& Hayes, 2011)

Apesar da área já ter adquirido expressiva disseminação e visibilidade no que tange à sua produção científica mundial, no Brasil este ainda é um campo incipiente. Dessa forma, o objetivo deste relato é divulgar e descrever dois eventos científicos conjugados sobre meditação/mindfulness ocorridos no ano de 2014 em São Paulo, Brasil, dos quais tive a oportunidade de participar na qualidade de mediadora, palestrante e ouvinte. Além de uma contextualização e caracterização geral dos eventos, serão explorados alguns temas apresentados, em especial aqueles com relevância para a ciência psicológica. Conforme já foi apresentado, é importante reforçar que o termo "meditação" pode ser utilizado como um "guarda-chuva" para descrever tanto a meditação concentrativa, quanto do tipo mindfulness (assim como outros tipos, tal como guiada e em movimento). Por isso, os termos meditação e mindfulness foram utilizados intercambiavelmente por diferentes palestrantes. No entanto, no presente artigo, ao longo do relato das apresentações, será sempre pontuado quando a meditação não for do tipo mindfulness. Além disso, quando apenas o termo meditação for utilizado, este estará sendo empregado no seu sentido genérico, referindo-se às diferentes práticas investigadas e relatadas.

\section{Sobre os Eventos}

Dois eventos interligados e subsequentes, cada um com duração de dois dias, aconteceram nas dependências do Teatro Marcos Lindenberg, na Universidade Federal de São Paulo (UNIFESP), entre as datas de 14 e 17 de Maio de 2014: o Pré-Simpósio "II Encontro de Mindfulness e Promoção da Saúde", presidido pelo
Prof. Dr. Marcelo Demarzo, com a colaboração dos demais integrantes da Iniciativa Mindfulness (que podem ser acessados em http:// encontromindfulness.com); e o " $4^{\circ}$ Simpósio Internacional de Medicinas Tradicionais e Práticas Contemplativas", promovido pela UNIFESP e Associação Palas Athena, e coordenado pelos Profs. Drs. Luiz Eugenio Araujo de Moraes Mello, Elisa Harumi Kozasa, Lia Diskin, entre outros (que podem ser conferidos em Anais do $4^{\circ}$ Simpósio Internacional de Medicinas Tradicionais e Práticas Contemplativas, 2014).

No Pré-Simpósio, os trabalhos abordados foram principalmente sobre a meditação mindfulness, enquanto no Simpósio as apresentações abarcaram temáticas sobre diferentes tipos de meditação, espiritualidade, bem como outras práticas consideradas integrativas, por exemplo, a medicina tradicional chinesa. Na qualidade de evento científico, ambos os simpósios destinaram-se a explorar e apresentar a produção científica nacional e internacional, tanto do ponto de vista da ciência básica, quanto aplicada, especialmente no que tange a contribuição das práticas à saúde e à sociedade.

\section{Palestrantes Nacionais e Internacionais}

O Pré-Simpósio trouxe conferencistas internacionais de referência no tema: Javier García-Campayo (Universidade de Zaragoza, Espanha), Joaquim Soler (Hospital de la Santa Creu i Sant Pau, Barcelona, Espanha), Yolanda López del Hoyo (Universidade de Zaragoza, Espanha), José Rivas (Universidade da República Oriental do Uruguai), Mauro Garcia Toro (Universidade das Ilhas Baleares, Palma de Mallorca, Espanha) e Caterina Vicens (Universidade das Ilhas Baleares, Palma de Mallorca, Espanha); e também contou com importantes palestrantes nacionais, tanto pesquisadores, como profissionais de diferentes esferas, dentre elas, saúde e educação: Elisa Harumi Kozasa (Instituto do Cérebro do Hospital Albert Einstein e Departamento de Psicobiologia da Escola Paulista de Medicina da Universidade Federal de São Paulo [EPM/UNIFESP]), Nelson Filice de Barros (Coordenador do GT Associação Brasileira de Saúde Coletiva 
[ABRASCO] de Práticas Integrativas e Complementares), Ana Regina Noto (Departamento de Psicobiologia, EPM/UNIFESP), Sandra Fortes (Centro de Mindfulness, Universidade Estadual do Rio de Janeiro), Júlio Lins (Coordenador do Núcleo de Apoio às Praticas Integrativas em Saúde da Prefeitura do Recife), Maria Cecilia de Mello e Souza (Programa Eicos, Universidade Federal do Rio de Janeiro), Regina Migliori (Instituto Migliori), Paulo Bloise (Núcleo Anthropos, UNIFESP), Stephen Little (Centro de Vivência em Atenção Plena, São Paulo) e Marcelo Demarzo (Centro Mente Aberta, EPM/ UNIFESP).

Seguindo o mesmo critério de relevância da atuação profissional dos palestrantes, o Simpósio contou com a presença internacional de Jamyang Dolma (Tibetan Medical Institute, India), Susan Andrews (Instituto Visão Futuro), Geshe Lobsang Tenzin Negi (Emory University, EUA) e Javier Garcia-Campayo (Universidad de Zaragoza, Espanha), assim como a participação de profissionais que vêm desenvolvendo trabalhos no cenário nacional: Luiza Hiromi Tanaka (UNIFESP), Eliana Rodrigues (UNIFESP), Diego Ladéia (UNIFESP), Marcelo Demarzo (UNIFESP), Carolina B. Menezes (Universidade Federal de Pelotas), Ricardo Monezi (Pontifícia Universidade Católica de São Paulo [PUC-SP]), Júlio Pérez (Universidade de São Paulo e Hospital Pérola Byington), Carol Ferreira de Andrade (UNIFESP), Lúcia Helena Rangel (PUC-SP) e Tiago Pires de Campos (Área Técnica de Práticas Integrativas e Complementares do Ministério da Saúde).

\section{Atividades Programadas}

No Pré-Simpósio, as atividades estiveram principalmente concentradas em 3 mesas redondas, estando um turno reservado para uma reunião do Grupo de Pesquisa "Mente Aberta", do Centro Brasileiro de Mindfulness e Promoção da Saúde, UNIFESP. Também aconteceu o lançamento de um livro em espanhol, o qual será traduzido para o português como "Mindfulness e Ciência: Da Tradição à Modernidade", organizado por Javier García-Campayo e Marcelo Demarzo. As atividades ainda incluíram um espaço dedicado à apresentação de trabalhos de Mestrado ou Doutorado previamente selecionados.

O Simpósio contou com cinco mesas redondas, sendo que em ambos os dias a primeira hora da tarde foi destinada à sessão de pôsteres previamente submetidos. Além disso, ambos os eventos ofereceram atividades práticas em torno dos temas nucleares, tal como técnicas meditativas, as quais foram guiadas por equipe de profissionais especializados. O público de ambos os eventos compreendeu professores, pesquisadores, estudantes de graduação e pós-graduação, representantes de Secretarias da Saúde, de organizações não governamentais (ONGs), bem como profissionais de diferentes áreas de atuação.

Destaca-se que todos os resumos das palestras e dos pôsteres apresentados em ambos os eventos estão publicados como Suplemento Especial intitulado "Anais do $4^{\circ}$ Simpósio Internacional De Medicinas Tradicionais e Práticas Contemplativas", na Revista de Pesquisa e Inovação Farmacêutica, volume 6, número 1, 2014. Estes são de livre acesso, podendo ser recuperados em http://pgsskroton.com.br/seer//index. $\mathrm{php} / \mathrm{RPInF} / \mathrm{article} / \mathrm{view} / 55$.

\section{Temas Abordados em Mesas Redondas}

A motivação para divulgar e descrever estes eventos pode ser em grande parte atribuída à abrangência dos trabalhos apresentados e sua pertinência para os campos da psicologia e da saúde em geral, assim como para o avanço do estudo da meditação nestes contextos, tanto em nível nacional, como internacional. Por exemplo, Joaquim Soler discutiu sobre a importância da "Mensuração Psicométrica de Mindfulness", salientando que escalas confiáveis para medir mindfulness são fundamentais para o avanço da área, pois permitem estudar mecanismos da prática, determinar que capacidades são especialmente desenvolvidas, discriminar pessoas com e sem patologia, bem como discriminar praticantes de não praticantes. No entanto, Soler ressalta que o conceito ainda é ambíguo, uma vez que diferentes definições operacionais medem componentes distintos e divergem com relação à condição uni ou multifatorial do construto. 
Marcelo Demarzo apresentou "Diretrizes para a Implementação de Mindfulness em Sistemas Sanitários", destacando que apesar de mindfulness já ser formalmente incluída como prática do Sistema Nacional de Saúde em locais como Inglaterra, a mesma configura uma intervenção complexa em saúde, exigindo alguns cuidados. Neste sentido, Demarzo salientou a importância de considerar-se os seguintes aspectos para o planejamento e execução deste tipo de implementação: plano e trabalho coordenados que permitam a variabilidade de modelos de intervenção (protocolos devidamente adaptados e voltados a atingir mecanismos e populações específicas); estudo piloto e de viabilidade (a fim de avaliar barreiras, facilitadores, aderência); modelo teórico norteador consistente; dosagem do volume de prática (inicialmente oferecer por tempos mais curtos e breves, visando atingir maior volume de pessoas); programas stepped-care (passo-a-passo distinto para diferentes demandas e gravidades); capacitação e qualificação profissional; avaliação do processo (abordagem qualitativa); avaliação de custo-efetividade; e observância de ausência de resultados (possivelmente indicativos de barreiras e resistências).

Em complemento a esta palestra, Júlio Lins e Sandra Fortes apresentaram um "Relato de Experiência de Implementação de Mindfulness para Pacientes e para Profissionais na Atenção Básica". Lins relatou uma experiência positiva com pacientes, destacando a alta aceitação e adesão aos grupos de práticas integrativas oferecidos em uma Unidade Básica de Saúde em Recife. Observou que neste contexto o nome da atividade deve ser facilmente assimilado pela comunidade, já que mindfulness pode ser um termo de difícil compreensão. Lins citou o exemplo do grupo que coordena, o qual chamou de "Programa Atentamente". Segundo o palestrante, este nome foi facilmente compreendido por todos os participantes. Também destacou a importância do uso de metáforas para transmitir conceitos chave sobre a prática, e observou que recomendar práticas breves, tal como 5 minutos três vezes por semana, facilitou que os participantes não criassem resistência em manter a prá- tica. Além disso, constatou que estipulando um tempo mínimo curto, os participantes acabavam fazendo a prática por mais tempo.

Por outro lado, Sandra Fortes apresentou as dificuldades vivenciadas na tentativa de inserir grupos de mindfulness com profissionais do Sistema Único de Saúde (SUS) no Rio de Janeiro. Dentre os principais desafios, Fortes destacou os problemas existentes na própria Unidade, os conflitos entre equipes de profissionais, a frequente troca e substituição de profissionais, as más condições das salas, a indisponibilidade dos profissionais em comparecer aos encontros, a alta rotatividade de participantes a cada encontro, e a não aceitação da duração de duas horas por encontro. A partir destas dificuldades, a palestrante discutiu alternativas para a implementação de mindfulness com profissionais, tal como encontros realizados em local distinto da Unidade; grupos integrados, combinando profissionais e pacientes; grupos de duração mais curta; e um planejamento de práticas e técnicas mais específicas e adaptadas à realidade do grupo. Lins e Fortes também destacaram que a forte religiosidade presente nas comunidades pode ser um desafio para a aceitação e adesão aos grupos de práticas integrativas.

Em outra palestra bastante pertinente, Stephen Little discorreu sobre a "Capacitação Profissional em Mindfulness", especialmente no contexto dos programas baseados em mindfulness, tal como o Programa de Redução de Estresse Baseado em Mindfulness (Mindfulness-Based Stress Reduction - MBSR) ou a Terapia Cognitiva Baseada em Mindfulness (Mindfulness-Based Cognitive Therapy - MBCT). Para trabalhar com estes programas, Stephen salientou que a capacitação completa deve abranger quatro passos: participação em um programa de oito semanas, para que inicialmente o profissional tenha a experiência de participar como paciente; prática diária, tendo em vista que mindfulness não deve compreender apenas uma técnica em um dado momento, mas um processo em que a regularidade da técnica possibilite experiências de atenção plena; formação para coordenar grupos de pacientes certificada por local reconhecido; e 
formação para coordenar grupos de capacitação profissional (multiplicação) certificada por local reconhecido.

Outro tema abordado e atual diz respeito aos "Dispositivos Eletrônicos para o Ensino e o Uso de Mindfulness". Após percorrer por uma breve revisão sobre o atual cenário de aplicativos de programas de saúde, discutindo a abrangência da indústria de smartphones no mercado global, Yolanda del Hoyo apresentou o aplicativo de mindfulness desenvolvido pelo seu grupo de pesquisa na Espanha. Além do objetivo geral de facilitar a aderência e a manutenção da prática, o aplicativo oferece uma série de ferramentas que facilitam e possibilitam a execução da prática, o entendimento prático e teórico de mindfulness, a autoavaliação da eficácia da prática pessoal, bem como a troca e a interação com outros usuários e praticantes.

Seguindo a linha de temas atuais, Geshe Lobsang Tenzin Negi falou sobre pesquisas recentes que têm investigado a eficácia da aplicação da meditação da compaixão em tratamentos médicos e psiquiátricos. A partir desta contextualização, Negi apresentou o Cognitive-Based Compassion Training (Treino Cognitivo de Compaixão), protocolo que desenvolveu a fim de possibilitar o treino cognitivo sistemático para o cultivo de sentimentos de compaixão e altruísmo. O mesmo é formado e conduzido a partir de oito passos descritos a seguir: desenvolver a atenção e a estabilidade da mente através de treinos da atenção focada; estimular e desenvolver insight acerca da natureza da experiência mental; cultivar autocompaixão; desenvolver equanimidade; desenvolver apreciação e gratidão; desenvolver afeto e empatia; exercitar a aspiração à compaixão; e exercitar a compaixão ativa. Durante o programa são apresentados fundamentos teóricos e práticos que possibilitem a incorporação destas práticas na vida diária. Negi destacou que o treinamento foi inicialmente desenvolvido para estudantes universitários, a partir da constatação do significativo aumento de doenças mentais e suicídio nesta população. Contudo, atualmente o programa também tem sido aplicado em escolas e diferentes contextos de saúde, com pesquisas financiadas pelo Natio- nal Institute of Health - NIH (Instituto Nacional de Saúde, Estados Unidos).

Além destas temáticas, algumas apresentações contribuíram destacando possíveis interfaces que o estudo de mindfulness e das chamadas práticas integrativas possibilitam com campos afins. Por exemplo, Javier Garcia-Campayo destacou a "Relação de Mindfulness com Psicologia Positiva e Saúde", discutindo que existe uma sinergia entre estas áreas, evidenciada por suas premissas complementares - tal como noções de aceitação, autoconsciência, identificação de valores e compromisso social -, e objetivos comuns, por exemplo, a busca de maior bem-estar psicológico. Garcia-Campayo enfatizou principalmente o quanto as técnicas de mindfulness podem auxiliar o praticante na ampliação da consciência de seus próprios processos, favorecendo o trabalho da Psicologia Positiva com relação à identificação de valores, forças e virtudes pessoais. Estes aspectos psicológicos, por sua vez, são considerados fundamentais para a obtenção ou manutenção de maior saúde física.

Durante a palestra "Efeitos da Meditação Focada na Regulação das Emoções e da Atenção", proferida pela autora do presente relato, foi proposto como o estudo da meditação do tipo concentrativa, concebida enquanto técnica de treinamento cognitivo, pode contribuir para o conhecimento básico e aplicado de Psicologia Cognitiva e vice-versa. Foram apresentados os resultados da avaliação de uma intervenção de seis semanas de meditação focada a partir de um paradigma cognitivo experimental. Os achados indicaram que a meditação focada ajudou os participantes - amostra não clínica de universitários - a diminuírem a interferência emocional de imagens desagradáveis durante uma tarefa computadorizada de atenção. Além disso, os participantes apresentaram uma melhora da atenção executiva, conforme evidenciado pela análise do índice de viés de resposta da Teoria de Detecção de Sinais. A autora ainda destacou que estes efeitos foram significativamente diferentes do grupo controle de relaxamento progressivo.

Elisa Kozasa estabeleceu uma relação entre "Meditação e Neurociência", demonstrando os efeitos que esta prática (considerando o tipo 
concentrativo e mindfulness) pode promover na atividade neural, tanto do ponto de vista estrutural, como funcional. A palestrante iniciou sua apresentação enfatizando que no contexto tradicional, meditação é lembrar-se de estar atento ao momento presente. Assim, a capacidade atentiva está na base do aprimoramento de muitas outras capacidades mentais, sejam elas cognitivas, ou emocionais. Kozasa prosseguiu apresentando os estudos que realiza juntamente a um grupo de pesquisa do Hospital Albert Einstein, em São Paulo, os quais demonstram que de fato a meditação regular pode produzir um incremente da cognição, tal como uma maior eficiência do processamento atencional. Por exemplo, a palestrante citou um estudo em que sua equipe observou que pessoas que realizaram um teste de atenção sustentada durante um exame de ressonância magnética funcional, e que não meditavam com regularidade, precisaram ativar mais áreas cerebrais e com maior intensidade para obter uma performance no teste equivalente à performance das pessoas que meditavam regularmente. Kozasa discutiu que este dado pode significar que as pessoas mais experientes em meditação têm um cérebro mais eficiente nesse tipo de teste de atenção, já que o treino atencional está na base do treino meditativo. No entanto, Kozasa alertou que a magnitude dos ganhos está diretamente relacionada à continuidade e à frequência de prática.

Outras falas abordaram como a meditação também pode ser utilizada e investigada em combinação com diferentes abordagens psicoterápicas já existentes, tal como as Terapias de Aceitação e Compromisso (ACT) e Dialética Comportamental (DBT). Com relação à ACT, Jose Antonio Guallar Claver discutiu, por exemplo, como o componente de aceitação de mindfulness pode constituir uma valiosa ferramenta para trabalhar com condições que envolvem algum tipo de evitação experiencial, tal como na dor crônica. Com base neste entendimento, apresentou resultados de um ensaio randomizado que o seu grupo de pesquisa conduziu a fim de testar a eficácia de ACT em pacientes com fibromialgia. Os resultados indicaram que a ACT foi mais eficaz do que o tratamento farmacológico usual para a redução da dor, sendo que os efeitos posi- tivos continuaram sendo observados seis meses depois do término da intervenção.

Referente à DBT, cujo tratamento é especialmente voltado para pacientes com transtorno de personalidade borderline, Joaquim Soler exibiu noções de porque minfulness é uma das habilidades treinadas neste protocolo, e porque possui alto potencial de produzir benefícios para este tipo de paciente. Além de auxiliar na regulação atencional e na consciência corporal, acredita-se que mindfulness tem o potencial de contribuir na diminuição dos níveis de impulsividade. De fato, um estudo realizado por Soler e seu grupo de pesquisa apoia esta hipótese; foi observado que pacientes borderline que receberam uma intervenção de 10 sessões de DBT com ênfase em habilidades de mindfulness apresentaram uma redução significativa em algumas variáveis específicas de impulsividade (relacionada à percepção do tempo e impulsividade planejada), em comparação a pacientes que receberam 10 sessões de DBT com ênfase na efetividade interpessoal, mesmo que uma melhora global da impulsividade tenha sido observada para ambos os grupos.

Ainda, a meditação foi discutida em relação aos contextos da Antropologia e da Educação. Concernente à perspectiva antropológica, Cecília de Mello e Souza salientou a potencial contribuição das "Abordagens Qualitativas em Mindfulness", tal como a etnografia, para o estudo e o entendimento da fenomenologia desta experiência. Os métodos qualitativos, segundo a palestrante, podem favorecer um enfoque mais processual e integral acerca do fenômeno mindfulness, contemplando os seus aspectos filosóficos, culturais e contextuais tanto tradicionais, como contemporâneos. Assim, Souza concluiu que estudar práticas meditativas a partir do paradigma da complexidade e de uma abordagem transdisciplinar de "mente-corpo-meio ambiente" pode complementar pesquisas experimentais, ajudando a gerar novos problemas de pesquisa.

Já Regina Migliori convidou a audiência a refletir sobre a interface entre "Práticas Integrativas e Contemplativas e Educação", defendendo que questões sobre desenvolvimento humano e saúde também devem perpassar o campo da edu- 
cação, caracterizando a tônica de ações preventivas e criativas neste contexto. Segundo Regina, este tipo de prática pode auxiliar no trabalho educativo sobre valores humanos e universais, valores sociais e valores pessoais, assim como no desenvolvimento de potencialidades humanas. No entanto, Migliori enfatizou a importância das práticas serem incorporadas à educação por meio de projetos e iniciativas pedagógicas bem planejadas e delineadas.

\section{Temas Abordados em Pôsteres}

Diversas pesquisas em andamento ou concluídas, de estudantes de graduação ou pós graduação, também foram apresentadas por meio de pôsteres. Por exemplo, Isabel Weiss Souza e colaboradores apresentaram um estudo de caso oriundo de um estudo piloto realizado para testar o uso de uma intervenção chamada Prevenção de Recaída Baseada em Mindfulness (Mindfulness-Based Relapse Prevention - MBRP) com pessoas fazendo tratamento para largar o tabagismo. Seguindo uma metodologia de ensaio clínico randomizado e controlado, com seguimento de 12 meses, os autores encontraram que após um ano do treino o participante continuava abstinente do tabaco, também apresentando mudanças de estilo de vida, tal como redução do consumo de álcool e perda de $15 \mathrm{~kg}$. Os autores destacaram desafios observados, tal como a dificuldade de manter a prática na rotina, assim como de realizar tarefas de casa.

Nesta mesma direção, Ana Regina Noto e colaboradores descreveram o processo inicial de negociação com gestores e equipes da atenção básica para a implementação do MBRP em uma Unidade Básica de Saúde no interior de São Paulo para mulheres em uso crônico de benzodiazepínicos. Apesar da vasta aceitação e interesse de todos os entrevistados, observou-se resistência por parte de alguns médicos, os quais demonstraram descrença na proposta. Foi constatado que a equipe de enfermagem e os Agentes Comunitários podem ser elementos chave no processo de recrutamento da população. Ainda, alguns profissionais sugeriram o uso de um termo alternativo, já que a palavra meditação poderia gerar resistência na comunidade. A intervenção foi então nomeada "Calma Mente". Por fim, grande parte das mulheres convidadas aceitou participar das práticas. Foi concluído que é viável implementar o MBRP na Unidade em questão, mas a sensibilização da equipe de saúde deve integrar o processo de implementação desde a fase de planejamento.

Alguns autores também avaliaram como níveis de mindfulness disposicional influenciam a relação entre estresse percebido e bem estar subjetivo em profissionais da atenção primária à saúde. Ana Atanes e colaboradores encontraram que médicos e enfermeiros apresentaram piores níveis de mindfulness e estresse percebido em comparação às outras categorias profissionais. Além disso, foi observado que menores níveis de mindfulness relacionaram-se com maiores níveis de estresse percebido. Enfermeiros e Agentes Comunitários de Saúde (ACS) apresentaram menores índices de afeto positivo, sendo que o grupo de ACS também apresentou um pior índice de satisfação de vida. Os autores discutem a utilidade de trabalhos que visem desenvolver habilidades de mindfulness nas redes de atenção primária à saúde.

Outro trabalho apresentado referiu-se ao uso de mindfulness para pacientes internadas com transtornos alimentares. Katya Stübing e demais autores relataram os achados de um estudo piloto em que um treinamento de 10 sessões semanais de mindfulness foi oferecido a pacientes com baixo peso. As dez participantes apresentaram melhora significativa nas escalas de depressão, ansiedade, flexibilidade cognitiva, transtornos alimentares e mindfulness disposicional. Não foram encontrados resultados significativos para ruminação. O estudo também demonstrou que as participantes com maior severidade apresentaram um maior engajamento nas práticas, assim como uma maior redução da ansiedade em comparação às participantes com menor engajamento. A partir destes resultados, os autores acreditam que um ensaio clínico completo é viável com esta população.

Já Renata Satelis e equipe relataram os resultados de um estudo que avaliou os efeitos de um programa (PROGRESS) de 8 semanas baseado nos princípios de mindfulness na redução de sintomas de ansiedade e depressão em trabalhadores de uma indústria. Observou-se que ao 
término da intervenção e em comparação aos 18 participantes do grupo controle (que não recebeu nenhuma atividade durante o período de oito semanas), os 23 participantes do programa apresentaram uma redução significativa dos sintomas de ansiedade e depressão, bem como um aumento dos escores de atenção plena, conforme medido pela escala de Atenção e Consciência Plena (Mindful Attention Awareness Scale - MAAS).

Assim como os estudos de intervenção, outra pesquisa apresentada bastante relevante à ciência psicológica diz respeito à validação da versão brasileira da Escala de Atenção e Consciência Plena (MAAS), cujo intuito é medir níveis de mindfulness disposicional. Além de traduzir e adaptar a escala à cultura brasileira, Vivian Barros e o seu grupo de pesquisa demonstraram que a versão brasileira apresentou bons índices psicométricos considerando a estrutura unidimensional da escala, evidenciados por um alfa de Cronbach de 0,83 e um índice teste-reteste de $.80(p<0,001)$. Além disso, conforme esperado, o estudo detectou que praticantes de meditação apresentaram escores maiores na escala em comparação a não meditadores. Também houve uma correlação significativa e positiva entre MAAS e uma escala de Bem-Estar Subjetivo, indicando uma adequada validade convergente.

Por fim, cabe relatar um estudo que investigou fatores de adesão à pratica diária em pessoas que participaram de treinamentos de mindfulness. Tânia Silva e colaboradores encontraram que entre os 75 respondentes, as técnicas de mindfulness mais frequentemente relatadas como prática diária foram as "práticas informais" (80\%), seguido pela "meditação da respiração" (57,4\%), sendo 10-15 minutos a duração média de prática diária. Muitos respondentes $(71,7 \%)$ relataram sentir dificuldades em manter práticas diárias, citando como principais causas a falta de disciplina e o alto número de tarefas diárias da rotina normal. Entre os fatores facilitadores da prática regular, fazer parte de um grupo de meditação foi citado como o principal. Alguns respondentes consideraram que receber lembretes para a prática diária por mensagens curtas de celular pode ser um facilitador potencial. Os autores concluem que todos estes aspectos deveriam ser levados em consideração no plane- jamento e na execução de treinos baseados em mindfulness.

\section{Considerações Finais}

É notória a expressividade que os estudos sobre práticas meditativas e integrativas vêm ganhando mundialmente, evidenciando estas temáticas como um campo potencial de atuação, pesquisa e articulação multiprofissional, interdisciplinar e metodológica.

No que diz respeito à ciência psicológica, os temas apresentados elucidam a estreita relação entre meditação e psicologia, tanto no que tange à convergência dos pressupostos teóricos subjacentes a ambos os campos, como à contribuição do estudo da meditação para a prática clínica e científica da psicologia. Neste sentido, destaca-se que a utilização de intervenções baseadas em meditação, tanto para grupos clínicos, como não clínicos, auxilia na ampliação da ciência psicológica aplicada, através de estudos de eficácia, fomentando a utilização de tratamentos comportamentais complementares em diversos contextos de saúde. No Brasil, em particular, as intervenções baseadas em meditação podem ser uma alternativa para ampliar a inserção da psicologia em diversos segmentos, tal como no Sistema Único de Saúde e em ambientes educacionais. Similarmente, o estudo da meditação pode favorecer o desenvolvimento da ciência psicológica básica, contribuindo para a compreensão de processos básicos como atenção, consciência, percepção, motivação, entre outros, bem como suas inter-relações, podendo ainda viabilizar e impulsionar investigações acerca da interface entre psicologia e neurociência. Além disso, através do estudo da meditação a Psicologia pode encontrar-se com muitas áreas afins, tal como as diversas áreas da saúde e das ciências biológicas, a educação, a antropologia, entre outras. Por outro lado, conhecimentos técnicos da Psicologia podem contribuir para a qualidade tanto da investigação científica, como da aplicação clínica da meditação. Como exemplos, respectivamente, pode-se citar o desenvolvimento de instrumentos psicométricos para estudar construtos relacionados à meditação, e a incorporação de técnicas terapêuticas estabelecidas, como 
algumas oriundas das Terapias Cognitivas, em programas baseados na meditação que visam promover desfechos psicológicos positivos em grupos clínicos específicos.

Portanto, entende-se que é importante e necessário divulgar e relatar este tipo de evento no cenário brasileiro, onde o estudo da meditação e os esforços para a sua aproximação com o campo da psicologia ainda são discretos e emergentes. De forma conjunta, as atividades desenvolvidas no Pré-Simpósio e Simpósio permitiram uma rica reflexão e discussão acerca de questões científicas e clínicas pertinentes e atuais, contribuindo para o crescimento, o reconhecimento e o avanço da área no Brasil. Cabe destacar que ainda é necessária uma discussão mais aprofundada sobre o emprego das diferentes terminologias, bem como a caracterização dos diferentes tipos de práticas ou protocolos. Por exemplo, houve pouco debate sobre como ou em que grau diferentes métodos e contextos (tal como meditação com foco, meditação sem foco, meditação em contexto religioso ou espiritual, meditação secular, etc) influenciam e/ou moderam os efeitos observados.

Mesmo que este não seja o único congresso que contempla o tema, espera-se que mais eventos desta natureza sejam criados e realizados em território nacional, especialmente visando que o estudo da meditação seja formalizado enquanto área da ciência psicológica Brasileira. Por fim, vale relatar que na condição de psicóloga e pesquisadora do tema da meditação, foi uma experiência emblemática poder participar destes eventos, pois além de tratarem de um campo de investigação e atuação ainda em franca expansão, oferecendo um panorama conceitual e científico abrangente para alunos e profissionais interessados no assunto, os mesmos configuraram um momento importante para o desenvolvimento da área.

\section{Referências}

Anais do $4^{\circ}$ Simpósio Internacional de Medicinas Tradicionais e Práticas Contemplativas. (2014). Revista de Pesquisa e Inovação Farmacêutica, 6(1, Supl. 1). Recuperado em http://pgsskroton. com.br/seer//index.php/RPInF/article/view/55
Cardoso, R., Souza, E., Camano, L., \& Leite, J. R. (2004). Meditation in health: An operational definition. Brain Research Protocols, 14, 58-60. doi:10.1016/j.brainresprot.2004.09.002

Chiesa, A., \& Malinowski, P. (2011). Mindfulness-based approaches: Are they all the same? Journal of Clinical Psychology, 67(4), 404-424. doi:10.1002/jclp.20776

Chiesa, A., \& Serretti, A. (2011). Mindfulness based cognitive therapy for psychiatric disorders: A systematic review and meta-analysis. Psychiatry Research, 187(3), 441-453. doi:10.1016/j. psychres.2010.08.011

Davis, D. M., \& Hayes, J. A. (2011). What are the benefits of mindfulness? A practice review of psychotherapy-related research. Psychotherapy, 48(2), 198-208. doi:10.1037/a0022062

Goyal, M., Singh, S., Sibinga, E. M. S., Gould, N. F., Rowland-Seymour, A., Sharma, R., \& Haythornthwaite, J. A. (2014). Meditation programs for psychological stress and well-being: A systematic review and meta-analysis. JAMA Internal Medicine, 174(3), 357-368. doi:10.1001/jamainternmed.2013.13018

Grossman, P., Niemannb, L., Schmidtc, S., \& Walachc, H. (2004). Mindfulness-based stress reduction and health benefits: A meta-analysis. Journal of Psychosomatic Research, 57, 35-43.

Lutz, A., Slagter, H. A., Dunne, J. D., \& Davidson, R. J. (2008). Attention regulation and monitoring in meditation. Trends in Cognitive Sciences, 12(4), 163-169. doi:10.1016/j.tics.2008.01.005

Menezes, C. B., \& Dell'Aglio, D. D. (2009). Os efeitos da meditação à luz da investigação científica em psicologia: Revisão de literatura. Psicologia: Ciência e Profissão, 29(2), 276-289. doi:10.1590/S1414-98932009000200006

Sedlmeier, P., Eberth, J., Schwarz, M., Zimmermann, D., Haarig, F., Jaeger, S., \& Kunze, S. (2012). The psychological effects of meditation: A meta-analysis. Psychological Bulletin, 138(6), 1139-1171. doi:10.1037/a0028168

Wallace, B. A., \& Shapiro, S. L. (2006). Mental balance and well-being: Building bridges between buddhism and western psychology. American Psychologist, 61(7), 690-701.

Recebido: $27 / 07 / 2015$

$1^{a}$ revisão: $27 / 01 / 2016$

Aceite final: 2/02/2016 